Christian Fandrych \& Maria Thurmair (1994):

Ein Interpretationsmodell für Nominalkomposita: linguistische und didaktische Überlegungen

Diese Publikation wurde zuerst veröffentlicht / This publication has been published first in:

Deutsch als Fremdsprache, 1, 34-44. 


\section{Ein Interpretationsmodell für Nominalkomposita: linguistische und didaktische Überlegungen}

\section{Einleitung}

Auf die Tatsache, wie relevant Wortbildung in der deutschen Sprache ist, wurde schon oft hingewiesen. Jeder Text des Deutschen, egal ob mündlich oder schriftlich, ob fachsprachlich oder alltagssprachlich, enthält eine Vielzahl von Wortbildungen verschiedenen Typs. Wortbildungen sind ganz allgemein ein ökonomisches und sehr produktives Mittel der Wortschatzerweiterung im Deutschen. Aber nicht nur die reine Frequenz von wortgebildeten Einheiten verbunden mit der Tatsache, daß aufgrund der hohen Produktivität von bestimmten Wortbildungsmustern diese Einheiten meist nicht im Lexikon zu finden sind, ist ein Argument für ihre Vermittlung im DaFUnterricht; vielmehr spricht auch die spezifische funktionale Qualität von Wortbildungen für eine Einbringung in eine pädagogische Grammatik. Wortbildungen stellen ein grundlegendes Verfahren zur Bildung neuer Bezeichnungen dar, sie dienen als wichtiges Mittel der Textstrukturierung und -konstituierung und darüber hinaus auch als bedeutsames Stilmittel; kurz: sie sind ein wesentliches Strukturmerkmal der deutschen Sprache.

Dabei zeichnen sich die jeweils (auch Wortart-)spezifischen Wortbildungsarten (Ableitung, Komposition, Konversion usw.) dadurch aus, daß sie textuell, kommunikativ, syntaktisch und wortbildungsimmanent unterschiedliche Funktionen wahrnehmen. Diese können hier nicht in ihrer ganzen Vielfalt dargestellt werden, vielmehr beschränken wir uns auf einen der häufigsten Wortbildungsprozesse überhaupt, die Komposition beim Nomen. Offenbar bietet Komposition die Möglichkeit, Relationen zwischen Entitäten mit der sprachlich unaufwendigsten Form zu kodieren, nämlich Engstellung (Kontaktstellung) und Reihenfolge. Es liegt auf der Hand, daß sich in dieser sprachlichen Elementarform vor allem solche Relationen zwischen Entitäten wiederfinden, die für die menschliche Lebenspraxis grundlegend sind.
Trotz der hohen Relevanz der Wortbildung für die deutsche Sprache wird sie aber in der Didaktik des Deutschen als Fremdsprache kaum thematisiert. Ein Blick in gängige Lehrwerke der Anfängerstufe bestätigt dies. Wenn überhaupt, werden vor allem Präfix- und Suffixbildungen behandelt, letztere meist nur im Zusammenhang mit Genus-Erschließungsregeln beim Nomen. Auch die Spezialliteratur zur Wortbildung im DaF-Unterricht umfaßt kaum mehr als ein Dutzend Beiträge. ${ }^{1}$ In der Wortschatzdidaktik und der Gedächtnispsychologie ist verschiedentlich dargelegt worden, ${ }^{2}$ daß Wörter besser im Feldzusammenhang gelernt werden und daß sie am besten zugänglich sind, wenn auf diese Weise möglichst viele Verknüpfungen hergestellt werden können. Vor diesem Hintergrund erstaunt es, daß im Rahmen solcher didaktischen Konzepte derjenige Bereich nicht berücksichtigt wurde, in dem Wörter schon sprachlich (formal wie semantisch) sichtbar in einen Zusammenhang gebracht werden, in dem offenbar auch elementare pragmatische Beziehungen der beteiligten Lexeme zueinander zum Ausdruck kommen.

Über ihre Funktion für die Wortschatzarbeit hinaus gewinnt die Beschäftigung mit Wortbildung im DaF-Unterricht u. E. aber auch Relevanz als Gegenstand sui generis. Dies ergibt sich aus der oben angedeuteten Strukturrelevanz von Wortbildung im Deutschen, die die Wortbildungskompetenz als Teil einer umfassenderen Sprachkompetenz erscheinen läßt. Vielfach in der Wortbildungsforschung thematisierte Phänomene wie Lexikalisierung, systematische Lücken und das Fehlen einer eineindeutigen Entsprechung zwischen semantischen Modellen und formalen Wortbildungsmitteln (vor allem in der Derivation) stehen einer Wortbildungsdidaktik grundsätzlich nicht entgegen und betreffen in unterschiedlichem Ausmaß produktive und rezeptive Kompetenz. So ist das Phänomen der Lexikalisierung bzw. der Normebene von Wortbildungen vor allem für 
die Rezeption problematisch, worauf unten noch einzugehen sein wird. Systematische Lükken dagegen sind nur beim Aufbau einer produktiven Kompetenz von Belang; die nicht vorhandenen 1:1-Entsprechungen von Modellen und formalen Mitteln betreffen weniger die Komposition als vielmehr die Suffigierung und die Präfigierung. Ziel einer Wortbildungsdidaktik, die durchaus schon im Anfängerunterricht einsetzen sollte, ist der Aufbau einer Wortbildungskompetenz, die den Lernenden dazu befähigt, sich die Bedeutung neu auftretender Wortbildungsstrukturen zu erschließen und Wortbildungen in Grenzen auch selbst zu produzieren.

Im folgenden wollen wir Ansätze der Wortbildungsforschung unter der Fragestellung ihrer Adäquatheit sowie ihrer Konsequenzen für eine pädagogische Grammatik prüfen, daran schließt sich als Hauptteil unserer Überlegungen ein eigenes Modell zur Interpretation von Nominalkomposita an, aus dem dann didaktische Konsequenzen und Hinweise für die konkrete Arbeit mit Nominalkomposita im DaF-Unterricht abgeleitet werden.

\section{Bedeutungsanalysen in der Wortbildungsfor- schung - ein kritischer Überblick}

Im Bereich der Nominalkomposition lassen sich im wesentlichen zwei Forschungsrichtungen unterscheiden:

a) Zur ersten Richtung zählen wir die syntaktisch orientierten Auffassungen, die besonders in den siebziger Jahren entwickelt wurden. Man operiert hier mit zugrundeliegenden Sätzen wie etwa: Waschmaschine: $\mathrm{x}$ wäscht mit der Maschine (= Prädikat-Adverbiale-Typ) $^{3}$ bzw: , die Maschine wäscht‘. Besonders im Bereich der Nomen + Nomen-Komposita wird in den syntaktischen Ansätzen oft mit sog. Proverben bzw. Relatoren gearbeitet, z. B. Fischfrau: ,Frau transferier Fisch'. 4

In jüngster Zeit wurden wortsyntaktische Ansätze entwickelt, ${ }^{5}$ die die verschiedenen Sprachebenen strikt trennen und modular beschreiben. Auch für die Syntax postulierte Prinzipien werden auf die Wortbildung angewandt, wenn auch etwas modifiziert bzw. „parametrisiert". Wortbildung gilt also als Kombinationsprozeß, der mit den gleichen universalgrammatischen Prinzipien operiert wie die Satzsyntax. Relevant sind dabei etwa Argu- mentvererbungsprinzipien oder Rekursivität. Diese Ansätze sind als relativ formal zu charakterisieren; der eigenständige, aber holistische Charakter der Wortbildung wird dabei eher verdeckt. Problematisch ist auch, daß Regellosigkeiten oder Unregelmäßigkeiten von diesen Modellen schlecht bzw. gar nicht erfaßt werden können.

Auch die materialreichen Untersuchungen des Instituts für deutsche Sprache sind im wesentlichen syntaktisch orientiert. ${ }^{6}$ Hier werden Nominalkomposita interpretiert nach der syntaktischen Rolle ihrer Konstituenten in entsprechenden Paraphrasen und den damit verbundenen semantischen Rollen. Insbesondere der letzte Ansatz ist zudem stark referenzsemantisch orientiert. Dies sowie die Detailliertheit mancher Klassifikationen, die mit der einen oder anderen Ausprägung von zugrundeliegenden syntaktischen Strukturen arbeiten, führen zu immer kleineren und feineren Unterteilungen der Wortbildungsmuster bzw. -typen, die trotzdem oft überbestimmt oder unterbestimmt sind. So sind etwa die Paraphrasen bei Ortner / Müller-Bollhagen ${ }^{7}$ insofern überbestimmt, als sie spezifische Kontextbedeutungen, die im „Rahmen“ der Wortbildungsbedeutung möglich sind, zu deren konstitutiver Bedeutungsbeschreibung erheben; sie sind gleichzeitig unterbestimmt, insofern sie andere, durch den Bedeutungsrahmen des Kompositums mögliche Kontextbedeutungen entweder ganz ausschließen oder auf andere Subtypen verteilen. Die Problematik dieses Ansatzes soll anhand eines Beispiels näher gezeigt werden.

Für das Kompositum Malerpinsel schlagen Ortner/Müller-Bollhagen ${ }^{8}$ etwa die Paraphrasen ,P., der von einem Maler verwendet wird“' ,P., den ein Maler verwendet /,P., mit dem ein Maler arbeitet"/,P. (, der) für den Maler (bestimmt/geeignet ist) vor. Obwohl mit mehreren nahezu synonymen Paraphrasen gearbeitet wird, die im übrigen teilweise unzutreffend sind (bleibt doch ein Malerpinsel auch dann ein solcher, wenn er nicht von einem Maler bzw. nicht zum Malen benutzt wird), läßt weder die Paraphrasierung noch die Benennung der semantischen Subklasse (,agentiv/ auktorial“), die offenbar lediglich durch die semantische Rolle des Erstgliedes motiviert ist, einen direkten Schluß auf die realisierte Grundrelation zu. Die Orientierung an der semantischen Rolle des Erstgliedes in einem angenommenen ,entsprechenden“ Satz führt 
dann dazu, Malerpinsel in eine Subgruppe mit Ponywagen, Orchesterkonzert und im übergeordneten Typ „agentiv/auktorial“ neben solche Bildungen wie Picasso-Bild, Polizeirazzia und Nazizeit zu stellen. Auf der anderen Seite erscheint ein Kompositum wie Bohrmaschine, das ganz offensichtlich dieselbe grundlegende semantische Relation zwischen den Konstituenten aufweist wie Malerpinsel, im Typ ,aktional" neben Bildungen wie Prosa-Schriftsteller, Kaffeegesellschaft, Aquarelltechnik und Bierabend. Hier werden offenbar Gemeinsamkeiten, die auch interpretatorisch relevant sind, vernachlässigt zugunsten einer dem Gegenstand inadäquaten Klassifizierungsgenauigkeit, die zudem an teilweise nur schwer nachvollziehbaren Kriterien orientiert ist.

b) Aus der Kritik an den überdeterminierenden syntaktisch orientierten Ansätzen kommen Coseriu ${ }^{9}$ oder Heringer ${ }^{10} \mathrm{zu}$ dem Schluß, daß Nominalkomposition nicht mit spezifischen Regeln $\mathrm{zu}$ beschreiben ist. Sie gehen davon aus, daß jedes Nominalkompositum eine nahezu unbegrenzte Anzahl von Interpretationen haben kann, die im konkreten Vorkommen dann von kontextuellen, situativen oder anderen pragmatischen Faktoren spezifiziert bzw. determiniert wird. Die einzige Regel, die nach diesen Ansätzen für die Interpretation eines Kompositums angegeben werden kann, ist, daß bei einem Kompositum AB „B etwas mit A zu tun hat".

Unter dem Blickwinkel der Didaktik sind beide Ansätze letztlich unbefriedigend. Die syntaktisch orientierten Ansätze legen nahe, daß dem Lernenden möglichst exakte Paraphrasen für Nominalkomposita vermittelt werden - und zwar sowohl syntaktisch als auch semantisch (über-)genaue Paraphrasen. Abhängig davon, welchen syntaktischen Typ der Paraphrase man wählt (mit Präpositionalattribut oder mit Satz), ergeben sich mehr oder weniger genaue und unterschiedlich viele Strukturen. Tatsächlich sind auch eine Reihe von vorgeschlagenen Übungstypen davon geprägt. ${ }^{11}$ Was die semantische Interpretation betrifft, die im Vordergrund stehen sollte, so wird auch diese in den wenigen Vorschlägen zur Didaktik über die Paraphrase erschlossen. Ziel dabei scheint zu sein, möglichst exakt die Unterschiede aufzuzeigen, etwa zwischen Mondflug und Chinaflug (,Flug zum Mond' vs. ,Flug nach China'). Geht man von dem zwei- ten Ansatz (b) aus, der als einzige generelle Regel für Komposita festhält, daß „B mit A etwas zu tun hat", die spezifischere Interpretation aber dem Kontext(-wissen), Situationswissen oder dem globalen Weltwissen überläßt, so sind daraus für die Didaktik im Grunde keine wortbildungsspezifischen Möglichkeiten mehr abzuleiten.

Kernproblem einer Didaktik von Nominalkomposita ist also zunächst die Bedeutungserschließung; an der Oberfläche formal nicht gekennzeichnete semantische Relationen müssen von Lernern erschlossen werden. Anders als Muttersprachler verfügen Fremdsprachenlerner hierbei im Regelfall nicht über ein gut ausgebautes mentales Lexikon, und die „Speicherung " frequenter Wortbildungseinheiten ${ }^{12}$, die Muttersprachlern einen direkten Zugriff auf die Bedeutung ermöglicht, steht Lernern im Regelfall noch nicht zur Verfügung. Aus diesem Grund kommt den Interpretationsstrategien (auch für usuelle Wortbildungseinheiten) ein zentraler Stellenwert zu.

Nun liefern die Ergebnisse der bisher dargestellten Ansätze der Wortbildungsforschung wenig Hilfestellung für eine solche Verstehensstrategie. Gerade die Auflösung von Nominalkomposita in syntaktisch aufwendige Paraphrasen verkompliziert eher noch den Verstehensprozeß, denn diese Paraphrasen sind selbst höchst fehleranfällig und rekurrieren zudem auf Sprach- und Weltwissen, das gerade bei Lernern noch nicht vorausgesetzt werden kann. Vielmehr muß für einen auch didaktisch fruchtbaren Interpretationsansatz gefordert werden, daß er zum einen möglichst weitgehend die im sprachlichen „Material“. (Wortbildungen im Kontext) vorliegenden (strukturellen, semantischen, textuellen) Hinweise zur Interpretation ausnützt, zum anderen eine begrenzte Zahl von recht allgemeinen und in der Anwendung breiten Interpretationsregeln bereitstellt, die in Fällen der Unterbestimmtheit des sprachlichen Materials selbst applikabel sind. So ließe sich ein Weg zwischen der Skylla der über-exakten Paraphrasen und der Charybdis des "everything goes" finden, auf dem rezeptive (und begrenzt produktive) Wortbildungskompetenzen vermittelt werden, die auch dem spezifischen Charakter der Nominalkomposition gerecht werden.

Wir wollen dies noch an einem Beispiel zeigen: Ein Kompositum wie Lernwiese ${ }^{13}$ würde bei den syntaktisch orientierten Ansätzen in 
mindestens zwei Strukturen aufgelöst, etwa: Wiese, auf der jemand etwas lernt und Wiese, an der jemand etwas lernt. Für die Ansätze, die die Bedeutungskonstitution wesentlich dem Kontext zuordnen, würde sich für eine rein lokale Interpretation lediglich ergeben: Wiese, die etwas mit Lernen zu tun hat. Unserer Meinung nach sind beide Ansätze mit ihren Paraphrasen inadäquat. Aus der nächstliegenden Analogie $\mathrm{zu}$ usuellen Wortbildungen heraus (etwa Arbeitszimmer) und aus einem Modell von elementaren Grundrelationen, das wir weiter unten noch explizieren, kann man die Bedeutung der Bildung als "Wiese“, die in einer Zweckrelation zu „Lernen“ steht, angeben. Die weitere Determination leistet der umgebende Kontext. Es zeigt sich, daß explizite syntaktische Paraphrasen (wie die beiden zuerst angeführten) die Komposita aus dem Kontext lösen und sie isoliert, rein lokal beschreiben. Damit nehmen sie im Extremfall lediglich die unzähligen Kontexte, die ein Kompositum näher determinieren können, in die Bedeutungsbeschreibung dieses Kompositums vollständig mit hinein.

Wie gezeigt, darf die Einbeziehung des Kontextes nun aber auch nicht dazu verleiten, in der Interpretation „B, das mit A zu tun hat“ $\mathrm{zu}$ verharren und alles andere dem Kontext zuzuschreiben. Es gibt durchaus Komposita, die bereits lokal recht weitgehend $\mathrm{zu}$ interpretieren sind. Sie sind dies in einem „mittleren Exaktheitsgrad“, der für die Kommunikation völlig $\mathrm{zu}$ genügen scheint. Gerade in dieser mittleren Exaktheit besteht die spezifische Qualität von Wortbildungen. Aufgabe einer pädagogischen Grammatik sollte es darum sein, Komposita mit den auf sprachlicher Ebene relativ generellen Bedeutungsrelationen $\mathrm{zu}$ beschreiben und diese mit den anderen Ebenen (der Normebene, dem Kontext) in Beziehung zu setzen.

An dieser Stelle ist an einige neuere Arbeiten zur Wortbildungsforschung, insbesondere zur Nominalkomposition, anzuschließen, die - unterschiedlich im Detail und auch im theoretischen Ansatz - zu einer differenzierteren Betrachtungsweise bezüglich der „Selbstexplikativität" bzw. der Kontext- und Wissensabhängigkeit der Interpretation von Nominalkomposita kommen. So machen Fanselow ${ }^{14}$ und Gersbach / Graf ${ }^{15}$ deutlich, daß sehr wohl Aussagen über auf sprachlich-semantischer Ebene bestehende Relationsfixierungen möglich sind, ohne diese von syntaktischen „Entsprechungen" herleiten zu müssen. Fanselow" ${ }^{16}$ unterscheidet etwa „relationale“ Nominalkomposita von solchen, bei denen die Relation aus einer der Konstituenten stereotyp abgeleitet werden kann und solchen, für deren Interpretation eine von vier "Grundrelationen" herangezogen werden kann. Kritisch anzumerken ist zu Fanselow insbesondere, daß er auch Herleitungen von Stereotyprelationen aus dem Erstglied zuläßt, eine Interpretationsrichtung, die zumindest aus der Sicht einer pädagogischen Grammatik nicht sinnvoll erscheint, wohl aber auch darüber hinaus Schwierigkeiten aufwirft, denn sie verkompliziert die Interpretationsmöglichkeiten unnötig und läuft dem Determinationsprinzip entgegen. Gersbach/Grafs Kritik an der Konzeption der Stereotyprelationen, sie seien letztlich außersprachlich, ${ }^{17}$ trifft im Kern die Frage nach dem sprachlichen Bedeutungsumfang von Nomina. Ohne hier näher darauf eingehen zu wollen, scheint uns das Stereotypkonzept aber tragfähig zu sein, wenn der Bedeutungsbegriff nicht $\mathrm{zu}$ weit ausgelegt wird und wenn man annimmt, daß die Interpretation vom Zweitglied ausgeht. Auch die bei Gersbach/Graf vorgenommene Unterscheidung in eine sprachlich-semantische (System-) Ebene und eine Normebene (Referenzfixierung im Sprachgebrauch) scheint uns fruchtbar zu sein; ein solches Modell ermöglicht es auch, spezifischer die unterschiedlichen LernerSchwierigkeiten im Bereich der Nominalkomposition $\mathrm{zu}$ differenzieren und $\mathrm{zu}$ hierarchisieren (vgl. dazu näher 3.3-3.5).

\section{Interpretationsmodell für Nominalkomposita}

Grundvoraussetzung für die Interpretation eines Kompositums ist natürlich die Kenntnis der Bedeutung der Bestandteile sowie die Fähigkeit, die Determinans-Determinatum-Strukturen von Komposita analysieren zu können. Hinzu kommt Wissen über den generellen Wert bzw. die generelle Qualität von Wortbildungen. Gemeint ist hier das Wissen, daß Wortbildungen einen ,begrifflichen Mehrwert" haben; mit Wortbildungen werden ja nicht völlig akzidentielle oder aber kommunikativ und pragmatisch unwichtige Relationen bezeichnet, sondern nur relevante. Das Wissen um den „begrifflichen Mehrwert“ von Wortbildung verhindert z. B., daß eine Wortbildung 
wie Arbeitszimmer angewendet würde auf ein Zimmer, in dem zufällig jemand arbeitet. Genauso erklärt der „begriffliche Mehrwert", warum es zwar ein Schlafzimmer, aber kein „Sitzzimmer" gibt.

In der Folge soll es darum gehen, ein vor allem für didaktische Zwecke funktionales Modell der Interpretation von Nominalkomposita vorzustellen, das zwar teilweise auf den schon genannten Ansätzen von Fanselow und Gersbach/Graf basiert, jedoch in Teilen anders verfährt. Insbesondere sind hier zwei Punkte vorzuklären: Zum einen impliziert unser Modell keine Verstehenshierarchien (vgl. hierzu näher 3.5), zum anderen fassen wir - im Unterschied etwa zur Wortsyntax ${ }^{18}$ - die Relationen nicht als Reflex der Valenz bzw. der Rektion eines Zweitgliedes, sondern als Reflex seiner semantisch-lexikalischen Eigenschaften auf. Insbesondere ist die Besetzungsflexibilität nominaler Leerstellen und die Art der „Ergänzungen" bei Nomina variabler als etwa bei Verben.

Im Unterschied zu Gersbach / Graf ${ }^{19}$ jedoch gehen wir davon aus, daß neben den ,selbstverständlichen Relationen" auch einige semantisch und pragmatisch relevante „Grundrelationen" die Interpretation von Komposita steuern können, die im sprachlichen und lebenspraktischen Alltagswissen von Hörern/ Sprechern verankert sind. Daß dies zum Teil an Außersprachlichem festzumachen ist, erscheint nur auf den ersten Blick als Widerspruch: Gerade der Grenzbereich zwischen sprachlichem, normbezogenem und pragmatischem Wissen ist für Fremdsprachenlerner oft am schwersten zugänglich. Dennoch scheint uns sicher, daß bei Komposita, die auf einer strikt sprachlichen Ebene uninterpretiert bleiben oder nur sehr vage interpretiert werden können, solche Wissensbestände Interpretationshierarchien nahelegen, die mitbeschrieben werden müssen. Hier scheinen gerade auch bei "modernen" Wortbildungsmustern sachlichsprachliche Analogien eine große Rolle $\mathrm{zu}$ spielen, die etwa zu Reihenbildung führen und mit schemaorientierten kognitiven Verarbeitungsprozeduren zusammenhängen. ${ }^{20}$ Lokal können Komposita entweder aus der Bedeutung ihrer Konstituenten heraus (3.1) oder mit Hilfe von Grundrelationen (3.2) erschlossen werden. In beiden Fällen kann diese Interpretation noch durch die Normebene spezifiziert werden (3.3).

\subsection{Lexikalisch-semantisch interpretierbaire Komposita}

Eine Reihe von Komposita läßt sich aufgrund der lexikalisch-semantischen Eigenschaften ihrer Konstituenten weitgehend interpretieren. Wir gehen hier von einem Bedeutungsbegriff aus, der stereotype Interpretationen mit angibt. So gehört es für uns etwa zu der Bedeutung des Wortes Kiste, daß sie aus einem festen Material besteht. Das heißt, zur Bedeutung eines Wortes rechnen wir nicht nur das Wissen um den Gebrauch eines Wortes, sondern auch grundlegendes, stereotypes Wissen um die mit einem Wort bezeichneten Entitäten. Bereits auf diese Weise lassen sich eine Menge von Komposita problemlos interpretieren. Ausgangspunkt für jegliche Art der Interpretation ist das Zweitglied. Dieses kann relativ unselbständig sein, ein relationales Nomen. ${ }^{21}$ Dann besetzt häufig die erste Konstituente die von der zweiten nahegelegte "Leerstelle“.

a) Relativ problemlos $\mathrm{zu}$ interpretieren sind solche Komposita, bei denen das Zweitglied deverbal und noch nicht begrifflich eigenständig geworden ist, etwa -ung-Ableitungen oder -er-Ableitungen vom Typ: Steuererstattung, Kindererziehung, Filmemacher, Obstverkäufer, Zigarrenraucher, Bücherleser, aber auch andere Deverbalia wie Reisebeginn. Wo die Ableitung wirklich semantisch eigenständig ist - wie etwa im Fall von Verkäufer - können andere, nicht direkt aus der Bedeutung des Zweitglieds erschließbare Relationen auftreten, vgl. Straßenverkäufer. Diese sind dann mit Hilfe der Grundrelationen interpretierbar (s. 3.2).

b) Eine vergleichbare Interpretation ergibt sich bei relationalen Nomina, die semantisch-logisch eine Bezugsgröße benötigen, wie Sohn oder Fan, oder auch Nomina wie Hälfte, Viertel. Damit lassen sich Komposita vom Typ Professorensohn, HardrockFan oder Kuchenhälfte, Käseviertel problemlos interpretieren.

c) Schließlich sind auf diese Weise auch Komposita mit solchen Zweitgliedern $\mathrm{zu}$ interpretieren, die eine Art lexikalische Leerstelle aufweisen, die im freien Gebrauch meist mit einem präpositionalen Anschluß gefüllt wird, wie etwa Angst (vor), Liebe (zu), Sehnsucht (nach). In diesem Sinne sind Komposita wie Prüfungsangst, Kinderliebe 
und Friedenssehnsucht eindeutig zu interpretieren.

Bei allen drei angeführten Gruppen wird mit dem Erstglied eine semantische Relation genutzt, die von der Bedeutung des Zweitglieds nahegelegt wird.

d) Etwas weniger deutlich ist diese Art der Interpretation bei Komposita wie Nagelfa$b r i k$, bei denen die erste Konstituente eine Leerstelle besetzt, die sich aus einer möglichen relationalen Lesart des Zweitglieds stereotyp ergibt. ${ }^{22}$ Für das angeführte Beispiel bedeutet das, daß zur Bedeutungsbeschreibung von Fabrik das Merkmal ,produziert etwas" gehört, womit die Relation zu Nagel festliegt. Auf ähnliche Weise können Weinladen, Operettentheater, Zeitungskiosk gedeutet werden. Das heißt, die Interpretation des Kompositums beruht auf Relationen, die eng mit dem Bedeutungskern des Zweitglieds verbunden sind. Der Übergang zu den Grundrelationen ist hier fließend, was sich auch daran zeigt, daß solche Bildungen häufig auch über Grundrelationen sinnvoll interpretiert werden können.

e) Ebenfalls lokal leicht interpretierbar sind Adjektiv-Nomen-Komposita wie Rundholz, da ihnen meist eine attributive Beziehung (verbunden mit dem „,begrifflichen Mehrwert") entspricht.

\subsection{Grundrelationen}

Die meisten anderen Komposita, deren Bedeutung nicht schon aufgrund der Semantik ihrer Konstituenten eindeutig erschlossen werden kann, sind mit Hilfe weniger Grundrelationen interpretierbar. Ausgehend von der Bedeutung des Zweitglieds und dessen lebenspraktischer Relevanz, werden zwischen den Konstituenten elementare Relationen hergestellt. Im Kompositum wird also die elementare pragmatische Relevanz von Gegenständen und Sachverhalten versprachlicht. Im wesentlichen ergeben sich hier drei zentrale semantische Grundrelationen und an diese anschließend drei weitere, weniger frequente Relationen. ${ }^{23}$

\section{SITUATION}

Das Zweitglied wird bezüglich des Erstgliedes raumzeitlich situiert, d.h., das Zweitglied steht in lokaler oder temporaler Relation zum Erstglied. ${ }^{24}$

In Abhängigkeit von der Bedeutung der Konstituenten und der spezifischeren Kontextde- termination werden hier typischerweise folgende Relationen realisiert (Ausgangspunkt ist immer das Zweitglied):

- 〈ist in〉 Stadtautobahn, Gartenbrunnen;

- 〈führt zu〉 Gartentür, Mondrakete, Urwaldexpedition;

- 〈stammt aus/von〉 Kalbsfilet, Erdöl, Fabriknagel

- 〈ist zum Zeitpunkt/im Zeitraum〉 Mittagessen, Abendkonzert.

In übertragenem Sinne situiert ist:

- 〈gehört an〉SPD-Vorstand, Gemeindewald.

\section{KONSTITUTION}

Das Zweitglied hat das Erstglied als wesentlichen, konstitutiven Bestandteil:

- 〈besteht ganz aus〉 Holztisch, Goldring, Glasflasche, Wollmantel, Leinensakko;

- 〈hat〉 Henkeltasse, Nußkuchen, Erdbeertorte, Giebelhaus;

- 〈in der Art (Form, Farbe ...) von〉 Würfelzucker, Zitronenfalter, Vogel-Strauß-Politik.

\section{ZWECK}

Das Zweitglied wird bezüglich seines Anwendungsbereichs (Erstglied) bestimmt:

- 〈dient zu〉 Nähmaschine, Arbeitstisch, Schlafzimmer, Malerpinsel, Schulranzen, Trainingshose, Kinderzimmer; hier finden sich besonders häufig Verb-Nomen-Komposita;

- 〈schützt vor〉 Schmerztablette, Hustensaft, Windjacke.

Weniger frequent und im Zusammenhang mit den ersten drei Relationen zu beschreiben sind die folgenden Relationen:

\section{1a) SITUATION-URHEBER}

Das Zweitglied wird bezüglich des Erstglieds näher situiert, wobei die (stammt aus/ von〉-Relation zusätzlich näher als 〈ist verursacht von > charakterisiert wird (Fabriknagel etwa zeigt den Übergang zwischen diesen beiden Relationen): Feuerschaden, Polizeirazzia, Brecht-Gedicht.

\section{2a) KONSTITUTION-THEMA}

Das Zweitglied hat das Erstglied als konstitutiven thematischen Bereich:

- 〈hat als Thema〉 Tierbuch, Friedenszeichen;

- 〈im Bereich〉 Erzählstil, Verkehrsministerium. 


\section{INSTRUMENT}

Das Zweitglied wird in seiner Funktionsweise durch das Erstglied charakterisiert:

- 〈funktioniert mit Hilfe von〉 Benzinmotor, Handbremse, Windmühle, Dampfkochtopf.

Die Instrument-Relation schließt an die Zweck-Relation insofern an und ist gegenüber dieser sekundär, als die Instrumentrelation immer schon einen Zweck voraussetzt; sprachlich wird dies etwa in Mehrfachkomposita deutlich; vgl. Dampf-kochtopf, Elektro-rollstuhl, wo kompositionell primär der Zweck und sekundär das „Instrument" versprachlicht wird. In anderen Fällen ist der Zweck dem Zweitglied lexikalisch inhärent oder pragmatisch deutlich. ${ }^{25}$

Mit diesen lokalen Interpretationsmechanismen werden Grundbedeutungen abgeleitet, die jedoch von der Normebene noch zusätzlich überformt werden können. Hierbei handelt es sich insbesondere um eine spezielle Referenzfixierung, die zu einer engeren bzw. modifizierten Lesart mancher Komposita führt.

\subsection{Normebene}

Auf der Normebene werden spezifischere usuelle Bedeutungen von Komposita festgelegt. Insbesondere tritt häufiger der Fall ein, daß die lexikalisch-semantisch oder mit Hilfe von Grundrelationen erschlossene Interpretation eines Kompositums zu weit oder vage ist; dann wird durch die Normebene die Bedeutung eingeengt oder verschoben. Das ist typischerweise der Fall bei mehr oder weniger stark lexikalisierten Nomina. Eine Bedeutungsverengung ergibt sich hier etwa bei Blaubeere oder Hochhaus. Lexikalisch-semantisch bedeutet Blaubeere ,Beere, die blau ist'. Die usuelle Bedeutung ist zwar enger, aber schon bei dieser rein lokalen Interpretation ergibt sich, daß weder Himbeeren noch Erdbeeren, noch Stachelbeeren gemeint sind. Die exakte Interpretation aber liefert die Normebene.

Schließlich kann auch der Fall auftreten, daß ein Kompositum rein lokal, also entweder aufgrund der lexikalisch-semantischen Bedeutung oder mit Hilfe der Grundrelationen, nicht eindeutig interpretiert werden kann. Auch in diesem Fall kann die Normebene die konventionelle Bedeutung festlegen. Für ein Beispiel wie das vieldiskutierte Fischfrau, für das Heringer über zehn verschiedene, für ihn offen- sichtlich gleichberechtigte Deutungen angibt ( „,Frau, die Fisch verkauft", ,Frau des Fischs“, ,Frau, die im Sternbild der Fische geboren", ,Frau und Fisch (= Nixe) ${ }^{6}$, Frau, die Fisch is $(\mathrm{s}) \mathrm{t}^{6}$, ,Frau, die Fisch produziert', ,Frau, die vom Fisch abstammt', ,Frau, die kühl wie ein Fisch ist", ,Frau, die den Fisch gebracht hat $\left.{ }^{6} . . . "\right)^{26}$ heißt das, daß aufgrund der Norm die konventionelle Bedeutung mit: ,Frau, die Fisch verkauft' anzugeben ist. Bei vielen Komposita setzt zwar das Sprachsystem einen relativ weiten Bedeutungsrahmen, innerhalb dessen verschiedene semantische Relationen möglich sind (von diesem Typ leiten Coseriu ${ }^{27}$ oder Heringer ${ }^{28}$ ihre - dann generalisierte - Interpretationsregel „A hat mit B zu tun“ ab); meist wird aber durch die Sprachnorm eine konventionelle Bedeutung - und das ist im Normalfall die pragmatisch nächstliegende - festgelegt.

Die im Sprachsystem oft angelegten unterschiedlichen Interpretationsmöglichkeiten sind also durchaus hierarchisch geordnet und keineswegs gleichberechtigt. Eine nicht der konventionellen Bedeutung entsprechende Interpretation muß deshalb auch immer durch einen höheren „Kontextualisierungsaufwand“ gesteuert werden (s. u.). Die konventionelle Interpretation, die durch die Normebene festgelegt wird, spiegelt oft bestimmte kulturelle Bedingungen oder Sichtweisen wider und kann somit auf allgemeinem kulturellem Wissen beruhen. Auf der Normebene können aber auch verschiedene „Subnormen“ zur Interpretation eines Kompositums relevant werden, die nicht allen Sprechern einer Sprachgemeinschaft bekannt sind. Dazu sind fachsprachliche Subnormen zu zählen, die in fachsprachlichen oder institutionalisierten Kontexten eine bestimmte, von der alltagssprachlichen (Standard-)Interpretation abweichende Deutung eines Kompositums festlegen.

In ähnlicher Weise kann auch bestimmtes gruppenspezifisches Wissen zu nicht notwendigerweise stabilen Subnormen führen, an denen nicht alle Sprecher teilhaben. Hier ist an die Verwendung von Komposita zu denken, deren adäquate Interpretation spezifische Wissensbestände voraussetzt, etwa das Wissen um gesellschaftliche Ereignisse oder ein bestimmtes episodisches Wissen. Dieses Wissen geht immer über den konkreten Einzeltext hinaus und verweist auf andere Texte oder Situationen. Im Extremfall kann dieses episodische 
Wissen auch von nur zwei Sprechern geteilt werden.

Bei der rein lokalen Interpretation und der Interpretation auf der Normebene spielt schließlich noch die Analogie eine wichtige Rolle. Bei einem Kompositum wie z. B. Bananenheizung, das im Kontext „Bananenheizung im Busch" in der Überschrift eines Zeitungsartikels auftrat, kann die erste, lokale Interpretation analog an zwei existente semantische $\mathrm{Mu}-$ ster (vgl. 3.4) anschließen: das Kompositum kann einmal in Analogie zu Ölheizung, zum anderen in Analogie zu Bananenrepublik interpretiert werden (wobei die zweite Interpretation sicher auch durch „im Busch“ gestützt wird). Die Analogie liegt „quer" zu den anderen Interpretationsverfahren; sie stellt Komposita in ein paradigmatisches Verhältnis zu anderen Komposita im Lexikon.

\subsection{Die Rolle des Kontextes}

Der Kontext interagiert insofern mit dem jeweiligen Kompositum, als er vor- und nachsteuernde Hinweise $\mathrm{zu}$ dessen Interpretation gibt; im Einzelfall kann der Kontext/die Situation auch lokale Uninterpretiertheit völlig kompensieren. Das kann für diejenigen Komposita gelten, denen weder lexikalisch-semantisch noch mit Hilfe der Grundrelationen eine spezifische Bedeutung zugewiesen werden kann, das heißt keine Bedeutung, die über „B hat mit A zu tun" hinausgeht und für die keine Normbedeutung existiert. Hier wird im wesentlichen die Interpretation von ad-hoc-Komposita festgelegt, so etwa bei dem in der Wortbildungsliteratur ausführlich diskutierten Beispiel Atomspatzen ${ }^{29}$ : Lexikalisch-semantisch oder mit Hilfe der Grundrelationen läßt sich dieses Kompositum nicht spezifischer deuten als ,Spatzen, die etwas mit Atom zu tun haben'. Eine konventionelle Bedeutung für dieses Kompositum existiert nicht. Somit kann auch auf der Normebene dem Kompositum keine spezifische Interpretation zugewiesen werden, so daß zur Deutung die kontextuellen Hinweise herangezogen werden müssen. ${ }^{30}$ Letztere werden aber auch dann relevant, wenn die lokal festgestellte oder auf der Normebene festgelegte konventionelle Deutung „uminterpretiert" wird, wenn etwa Hosenträger im Sinne von ,Person, die Hosen anhat" interpretiert wird. In beiden Fällen (Interpretationsfestlegung oder Uminterpretation) aber muß es im Kontext oder in der Situation genügend auffäl- lige Hinweise geben. Nicht standardmäßig erschließbare Relationen erfordern also einen erhöhten Textaufwand, vgl.:

Bananenheizung im Busch

Ingenieur will Rodung des ugandischen Tropenwaldes aufhalten.

Kampala (epd). Der ugandische Ingenieur George Kyazze empfiehlt seinen Landsleuten, künftig mit Bananen, Kaffee und Mehl zu heizen: Er produziert Briketts aus verkohlten Bananenschalen, Kaffeehülsen und Maniokmehl. George Kyazze will nicht tatenlos zusehen, wie die Tropenwälder seines ostafrikanischen Landes nach und nach vernichtet werden. Wissenschaftler an der Makerere Universität in der Hauptstadt Kampala fürchten, daß dem Land von der Größe der Bundesrepublik bald die Verwüstung droht, wenn die Zerstörung der Wälder mit der derzeitigen Geschwindigkeit weitergeht. ... (Süddeutsche Zeitung. 7.7.1990, S. 10, nach Lauterbach, aaO)

Beim ersten Auftreten des Kompositums wird aus der Kenntnis der Bedeutung der Bestandteile und mit Hilfe analoger Wortbildungen eine annähernde Interpretation der Art ,Heizung mit Bananen', ,Heizung für Bananen', ,Heizung, die nicht richtig funktioniert" (in Analogie zu der figurativen Bedeutung von Bananenrepublik ${ }^{31}$ ) entstehen. Die weiteren Hinweise im Text mit Bananen ... heizen, Briketts aus verkohlten Bananenschalen engen die Interpretationsmöglichkeiten auf eine Deutung ein.

Ein zusammenfassendes Interpretationsmodell für Komposita zeigt Abb. 1 .

\subsection{Verstehensprozesse}

Wortbildungserscheinungen treten in den seltensten Fällen kontextlos auf. Dieser von der Wortbildungsforschung immer wieder in den Mittelpunkt gestellte Fall ist außerhalb der Linguistik ein deutlich markiertes Phänomen. Wortbildungen begegnen vielmehr im Text, der wiederum in bestimmte Kommunikationssituationen eingebettet ist und auf Hörer-/ Sprecherwissen Bezug nimmt. Somit ergibt sich für Verstehensprozesse von Wortbildungen, daß eine nur von der lokalen Interpretation ausgehende Verstehensleistung ein Sonderfall ist. Vielmehr interagieren Kommunikationssituation, (Kon-)Text, Normebene und „lokale“ Interpretationen beim Aufbau der Bedeutung von Komposita. Wie sich diese Ebenen genau wechselseitig in natürlichen Kom- 
$A b b .1$

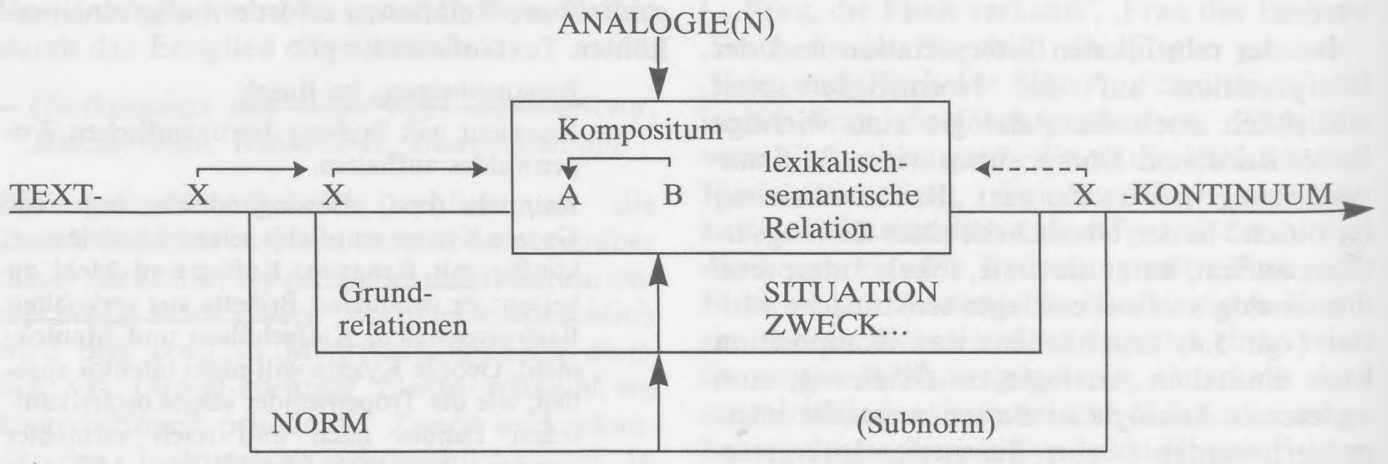

munikationssituationen unterstützen, darüber liegen uns für die Wortbildung keine empirischen Untersuchungen vor. Es scheint jedoch sicher zu sein, daß der (Kon-)Text durch bestimmte Signale wie das Auftreten bestimmter Lexeme und expliziter Relationen eine Erwartung an die Bedeutung des dann auftretenden Kompositums aufbaut bzw. vorsteuert. Eine Ausnahme stellen lediglich Überschriften dar, die aber als textuell markiert gelten müssen. Hier wird die Möglichkeit zur endgültigen Deutung eines in der Überschrift auftretenden Kompositums gewissermaßen suspendiert, bis der dazugehörige Text mit den entsprechenden Interpretationshinweisen rezipiert worden ist in dieser Hinsicht sind Komposita in Überschriften kataphorisch.

Außer im Spezialfall „Überschrift" wird die Interpretationserwartung durch Texthinweise vorgesteuert; diese Interpretationserwartung muß mit der lokalen Interpretationsebene und der Normebene, verrechnet' werden. Entsteht an einer Stelle des Interpretationsprozesses ein Widerspruch, muß eine der genannten Ebenen problematisiert werden, und es muß versucht werden, über alternative Interpretationen $\mathrm{zu}$ neuen, adäquateren Deutungen zu gelangen. Dies ist der Fall etwa bei Muttersprachlern, die eine bestimmte Subnorm nicht kennen (Fachsprache, episodisches Wissen) und daher die allgemeinsprachliche Norm der Interpretation zugrunde legen. Ähnlich verhält es sich bei der Fehlinterpretation des Vortextes, der lexikalisch-semantischen Relation oder der Heranziehung eines inadäquaten Analogieschlusses. Beim Fremdsprachenlerner können vor allem dadurch besondere Probleme entstehen, daß die Normebene defizitär ist - vorausgesetzt, daß der Vortext verstanden wurde, die
Bestandteile des Kompositums bekannt sind und der Lerner über Wortbildungsregularitäten verfügt.

\section{Didaktische Schlußfolgerungen}

Als didaktische Schlußfolgerung aus dem vorgestellten Modell läßt sich festhalten, daß im Unterricht sinnvollerweise vor allem das „lokale" Verstehen (lexikalisch-semantische Relationen und Grundrelationen) und die Strategien zur kontextgesteuerten Interpretation gefördert werden sollten. Die Normebene ist stärker idiosynkratisch. Grundvoraussetzung für den Aufbau einer Wortbildungskompetenz ist natürlich das Erkennen der DeterminansDeterminatum-Struktur eines Kompositums. Der erste Schritt der Vermittlung ist somit die Analyse eines Kompositums AB als ein ,bestimmtes B'. Gegenüberstellungen wie Mädchenschule vs. Schulmädchen, wie sie in manchen Lehrwerken zu finden sind, führen u. E. eher zu einer Verwirrung der Lerner und $\mathrm{zu}$ unterrichtsspezifischen Fehlern. ${ }^{32}$

Als Möglichkeit, das lokale Verstehen zu fördern, scheinen uns gerade auch gelenkt-produktive Übungen gut geeignet. Dabei kann es aber nicht darum gehen, beliebige Wörter wahllos kombinieren zu lassen, wie das oft in Kettenspielen geschieht. Vielmehr sollten pragmatisch relevante Relationen produktiv wie rezeptiv gefördert und gefestigt werden. Hier ließe sich etwa an Assoziogramme mit „Vorsteuerung" durch Angabe bestimmter relevanter Grundrelationen denken (vgl. Abb. 2).

Diese semantisch-funktional orientierte Art der Einübung von Wortbildungsregularitäten scheint uns wesentlich besser der spezifischen Qualität von Wortbildung zu entsprechen als die meist vorgeschlagenen syntaktischen Para- 

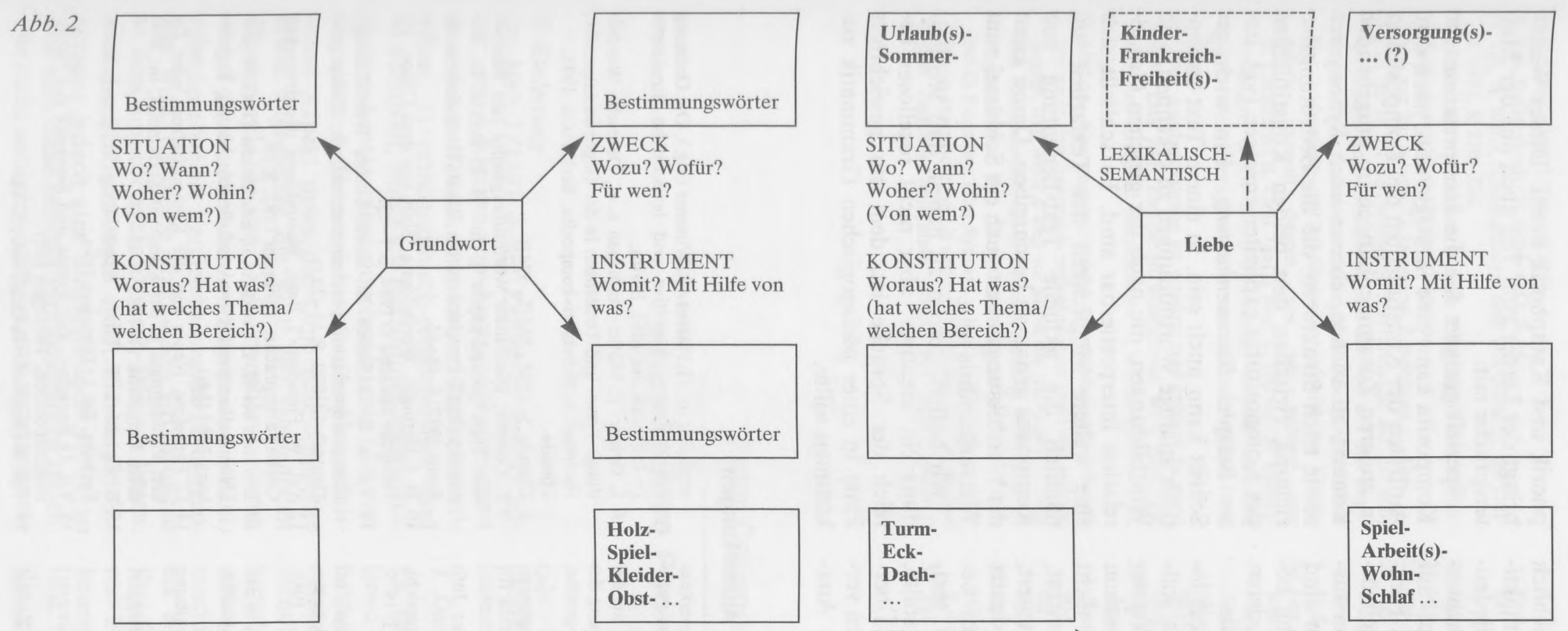

\section{SITUATION \\ Wo? Wann? \\ Woher? Wohin?}

(Von wem?)

KONSTITUTION

Woraus? Hat was?

(hat welches Thema

welchen Bereich?)

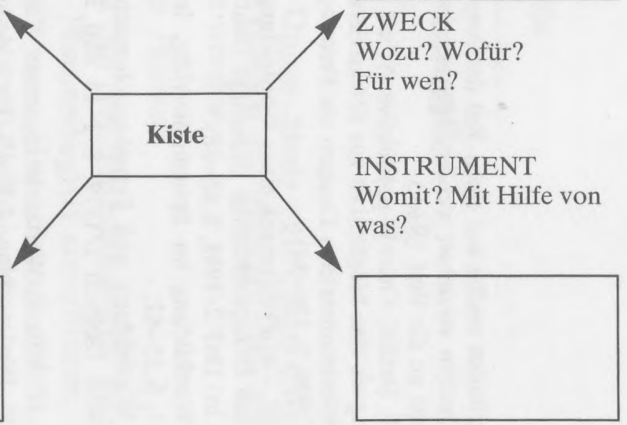

Wortschatzarbeit:

Truhe: Stein-

Schachtel: Papier-

Korb: Draht-

Weiden-
Papp(e)-

\section{SITUATION}

Wo? Wann?

Woher? Wohin?

(Von wem?)

\section{KONSTITUTION}

Woraus? Hat was?

(hat welches Thema/

welchen Bereich?)

\section{Erker- \\ Stuck-}

...

\section{Wortschatzarbeit:}

Saal ...

Raum ...

Stube ...

\section{ZWECK}

Wozu? Wofür?

Für wen?

\section{INSTRUMENT}

Womit? Mit Hilfe von was?

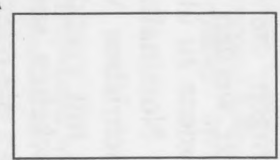


phrasenübungen, die oft sogar hauptsächlich dazu genutzt werden sollen, andere syntaktische Phänomene zu üben. ${ }^{33}$ Wenn also Bedeutungen von Nominalkomposita im Sprachunterricht beschrieben werden, sollte dies auf keinen Fall mit syntaktisch komplexen Paraphrasen geschehen, sondern mit Schlüsselwörtern, die etwa die relevante Grundrelation ausdrücken. Diese semantischen Relationen sind im übrigen ja nicht auf die Wortbildung beschränkt, sondern spielen auch in anderen sprachlichen Zusammenhängen eine Rolle.

Neben der Vermittlung der Fähigkeit zur lokalen Interpretation eines Kompositums sollten in einem zweiten Schritt textuelle Verstehensstrategien aufgebaut werden. Wie schon mehrfach betont, treten Wortbildungen nicht isoliert auf, sondern immer in einem Kontext, der ihre Interpretation wesentlich steuert, stützt und erleichtert. Für den Lerner bedeutet dies einmal allgemein, daß er über ein bestimmtes Grundwissen um textuelle bzw. textlinguistische Eigenschaften verfügen sollte, daß er z. B. die spezifische Funktion von Überschriften, den internen Aufbau von Texten verschiedener Textsorten, Phänomene wie Ana- phorik und Kataphorik kennt. Dieses Wissen bringt der Lerner zum Teil auch aus der Muttersprache mit.

Speziell geeignet für die Interpretation von Komposita kann eine Strategie sein, nach dem Auftreten der Konstituenten des Kompositums in anderen Zusammenhängen im umgebenden Kontext zu suchen, ebenso nach Synonymen sowie nach Strukturen, die die Bedeutungsbeziehung zwischen den beiden Konstituenten des Kompositums expliziter anzeigen (vgl. unser Beispiel Bananenheizung). Ein wichtiger Schritt kann auch sein, in einem Text semantisch analoge Wortbildungen $\mathrm{zu}$ erkennen, also Wortbildungen, die nach der gleichen Grundrelation interpretierbar sind. Es schließt sich eine weitere Möglichkeit der Textarbeit an, nämlich die aktuelle Text-Bedeutung von Komposita genau zu beschreiben. Damit kann die Wortbildungsarbeit auch ein Schlüssel zum Textverständnis sein.

Wir hoffen, gezeigt zu haben, daß Wortbildung ein kreativer, aber nicht regelloser Bereich der Sprache ist, dem ein gewichtiger Platz in einer pädagogischen Grammatik zukommen sollte.

\section{Anmerkungen}

* Aus Platzgründen mußte auf einen Teil der Anmerkungen bzw. Quellen verzichtet werden. Interessenten wenden sich bitte an die Verf. (Red.)

1 Vgl. u. a. T. Dreßel, Gelenkt-produktive Arbeit an der Wortbildung als eine Möglichkeit zur Effektivierung des Fortgeschrittenenunterrichts Deutsch als Fremdsprache, in: DaF 6/1986, S. 336-341;

W. Reinhardt, Zur Problematik eines Wortbildungsminimums für den fachsprachlichen Unterricht Deutsch für Ausländer, in: DaF 2/1988, S. 65-68;

R. Saxer, Wortbildung im Sprachunterricht, in: Info DaF 1/1991, S. 55-62;

G. Storch, Wortbildung und Fremdsprachenunterricht, in: Zielsprache Deutsch 2/1979, S. 2-13 und 3/1979, S. 2-12.

2 Vgl. z. B. H. Wilms, Wörter in unserem Kopf, in: M. Müller/L. Wertenschlag/J. Wolff (Hg.), Autonomes und partnerschaftliches Lernen, Berlin u. a. 1989, S. $145-160$.

3 Vgl. etwa G. Thiel, Die semantischen Beziehungen in den Substantivkomposita der deutschen Gegenwartssprache, in: Muttersprache 4/1973, S. 377-404.

4 Vgl. z. B. W. Kürschner, Zur syntaktischen Beschreibung deutscher Nominalkomposita, Tübingen 1974.

5 Vgl. etwa S. Olsen, Wortbildung im Deutschen, Stuttgart 1986;

J. Toman, Wortsyntax, Tübingen ${ }^{2} 1987$;

Kritisch dazu: C. Fandrych, Wortart, Wortbildungsart und kommunikative Funktion, Tübingen 1993;

W. Motsch, Semantische Grundlagen der Wortbildung, erscheint in: G. Harras / R. Wimmer (Hg.), Die Ordnung der Wörter - kognitive und lexikalische Strukturen (= Jahrbuch des IdS), Berlin.

6 L. Ortner/E. Müller-Bollhagen u. a., Deutsche Wortbildung: Typen und Tendenzen in der Gegenwartssprache, Hauptteil 4: Substantivkomposita, Berlin u. a. 1991.

7 Ebenda.

8 Ebenda, S. 138 f., $565 \mathrm{ff}$., $574 \mathrm{ff}$.

9 E. Coseriu, Inhaltliche Wortbildungslehre (am Beispiel des Typs "coupe-papier"), in: H.-E. Brekle/D. Kastovsky (Hg.), Perspektiven der Wortbildungsforschung, Bonn 1977, S. 48-61.

10 H. J. Heringer, Wortbildung: Sinn aus dem Chaos, in: Deutsche Sprache 1/1984, S. 1-13.

11 Vgl. z. B. G. Storch/M. Storch-Luche, Wortbildungsübungen im Fremdsprachenunterricht, in: Zielsprache Deutsch 1/1979, S. 11-23.

12 Vgl. W. Fleischer/I. Barz, Wortbildung der deutschen Gegenwartssprache, Tübingen 1992, S. 16.

13 Erwähnt bei H.-E. Brekle u.a., Endbericht DFG-Projekt „Nominalkomposita“ (= Arbeitsbericht Nr. 41), Regensburg 1984 (Ms).

14 G. Fanselow, Neues von der Kompositafront oder $\mathrm{Zu}$ drei Paradigmata in der Kompositagrammatik, in: Studium Linguistik 11/1981, S. 43-57.

15 B. Gersbach/R. Graf, Wortbildung in gesprochener Sprache, Bd. 1, Tübingen 1984.

16 Vgl. G. Fanselow, Zur Syntax und Semantik der Nominalkomposition, Tübingen 1981.

17 Vgl. B. Gersbach/R. Graf, aaO, S. 44. 
$18 \mathrm{Vgl}$. vor allem J. Toman, aaO.

19 Vgl. B. Gersbach/R. Graf, aaO, S. 44.

$20 \mathrm{Vgl}$. W. Wilss, Schematheorie und Wortbildung, in: DaF 4/1992, S. 230-234.

21 „Relational“ verstehen wir als Oberbegriff für semantisch ergänzungsbedürftige Nomina.

22 Vgl. zur Stereotypie G. Fanselow, Zur Syntax ..., aaO.

23 Mit der in 3.1 beschriebenen Interpretation auf der lexikalisch-semantischen Ebene und den von uns hier angesetzten Grundrelationen läßt sich der überwiegende Teil der in einschlägigen Publikationen aufgeführten Korporateile sinnvoll deuten, wie wir anhand von B. Gersbach/R. Graf $(\mathrm{aaO})$ und L. Ortner/E. MüllerBollhagen $(\mathrm{aaO})$ überprüft haben; deshalb stammen auch viele der Beispiele von Ortner/Müller-Bollhagen.

24 Diese Ausdrucksweise ist verkürzt und explizit zu lesen als: „Das vom Zweitglied Bezeichnete wird bezüglich des vom Erstglied Bezeichneten ...". Dies gilt auch im folgenden.

25 Mit den hier angenommenen Grundrelationen erheben wir nicht den Anspruch, vollständig zu sein; wir meinen jedoch, daß weite - und vor allem die relevanten - Teile des Kompositabestands damit abgedeckt sind. Der oft angenommene Typ „Kopulativkompositum“ etwa, dem bei G. Fanselow (Zur Syntax ..., aaO, S.215) die und-Relation entspricht, spielt unseres Erachtens im Deutschen eine denkbar marginale Rolle. Vgl. dazu genauer:
E. Breindl/M. Thurmair, Der Fürstbischof im Hosenrock. Eine Studie zu den nominalen Kopulativkomposita des Deutschen, in: Deutsche Sprache 1/1992, S. 32-61.

26 Vgl. H. J. Heringer, aaO, S. 2.

27 Vgl. E. Coseriu, aaO.

28 Vgl. H. J. Heringer, aaO.

29 Vgl. dazu W. Wildgen, Makroprozesse bei der Verwendung nominaler Ad-hoc-Komposita im Deutschen, in: Deutsche Sprache 3/1982, S. 237-257;

H. J. Heringer, aaO, S. 8.

30 Der entsprechende, bei W. Wildgen (aaO, S. 242) zitierte Kontext aus dem "Spiegel“ lautet: (1) Herr X entdeckte rings um das bayrische Atomkraftwerk Ohu zahlreiche Spatzen mit weißen Federn (2) die weißen Spatzen von Ohu (3) die Atomspatzen. Damit wird gleichzeitig eine SITUATION-URHEBER-Relation nahegelegt, vgl. ebd.

31 Vgl. dazu die Bedeutungsangabe in G. Wahrig, Deutsches Wörterbuch, München 1986, s.v. Bananenrepublik: „Land, das nahezu ausschließlich vom Südfrüchteexport lebt; 〈fig. > Land, in dem die wirtschaftl., politisch-moral. od. rechtl. Verhältnisse unsicher oder korrupt sind."

32 Vgl. J. Bahns, Schülerspezifische vs. entwicklungsspezifische Fehler, in: Linguistische Berichte 1/1986, S. 4-17.

33 Vgl. G. Storch/M. Storch-Luche, aaO, S. 13.

\section{Peter Colliander}

\section{Kontrastive Phonematik}

\section{Einleitung}

Im Heft 1/1993 dieser Zeitschrift schlug ich vor, mit 4 Klassen von Ausspracheproblemen zu rechnen: 1) artikulatorischen, 2) phonematischen, 3) orthographisch-orthoepischen und 4) auditiven, wobei ich nur auf den artikulatorischen Aspekt näher eingehen konnte. ${ }^{1}$ Im vorliegenden Aufsatz nehme ich den phonematischen Aspekt unter die Lupe und bespreche etwas allgemeiner die Rolle der segmentalen Phonematik für den DaF-Phonetikunterricht, wobei jedoch auch hier vorzugsweise die dänische Sprache als Vergleichssprache herangezogen wird, da meine praktischen Erfahrungen in dem Sprachenpaar Deutsch-Dänisch liegen. Für die Beobachtungen spielt die Vergleichssprache jedoch eine untergeordnete Rolle, so daß sich diese beliebig verallgemeinern lassen. Die nicht weniger wichtigen, auf suprasegmen- tale Unterschiede zurückzuführenden Probleme müssen aus Platzgründen außer acht gelassen werden.

\section{Das Phonem}

Es ist im Phonetikunterricht unumgänglich, einen Phonembegriff einzuführen. Ich spreche bewußt von „einem“ und nicht „dem“ Phonembegriff, denn ich möchte eine kleinere $\mathrm{Ab}$ wandlung des traditionellen Phonembegriffes vorschlagen, die m. E. die Klassenbildung, um die es ja geht, für die Studenten verständlicher macht. Traditionell definiert man ein Phonem als eine abstrakte Einheit, die sich durch ihre Funktion in der Sprache von allen anderen Einheiten unterscheidet: Laute, die nicht kommutieren, gehören ein und demselben Phonem an. Eine andere Möglichkeit wäre - für die pädagogische Situation -, eine konkrete 